


\title{
Toothbrush Abrasion of Resin Composites with Different Filler Concepts
}

\author{
Toshimitsu Suzuki, Hideaki Kyoizumi, Yasuyuki Araki, Werner J Finger, Masafumi Kanehira
}

\begin{abstract}
Purpose: To investigate the effect of tooth brushing wear with and without calcium carbonate slurry on four commercial resin composites based on different filler concepts.
\end{abstract}

Materials and methods: One nanofiller composite MI FIL (MFI, GC), one conventional hybrid type Venus (VEN) and two nanohybrid composites Venus Diamond (VED) and Venus Pearl (VEP) all from Heraeus Kulzer were examined. Forty beam-shaped specimens $(12 \times 3 \times 3 \mathrm{~mm})$ from each product were prepared and subdivided in two equal groups for pregrinding on $\mathrm{SiC}$ paper $\# 600$ and \#4000 respectively. Ten specimens of each pre-ground group were subjected to toothbrush abrasion with calcium carbonate slurry, and 10 to toothbrushing with water only. The samples were submitted to five subsequent runs of 10,000 brushing strokes. Depth of wear and surface roughness $(\mathrm{Ra})$ were measured with a profilometer after each $10 \mathrm{k}$ strokes. Specimens after $50 \mathrm{k}$ strokes were assessed by SEM. Additionally, the KHN $(0.25 \mathrm{~N} / 15 \mathrm{~s})$ and the degree of conversion (DC) were determined. Data analysis was done by linear regression and ANOVA followed by Tukey's post-hoc test $(p \leq 0.05)$.

Results: Toothbrushing with abrasive slurry produced significantly different wear depths: VEN > MIF > VEP > VED. Linear relationships between depth of wear and number of cycles $\left(r^{2} \geq 0.94\right)$ were established for each material. Pregrinding with $\mathrm{SiC} \# 4000$ resulted in less wear than pregrinding with \#600. Ra figures were much higher for VED than for the other materials tested. MIF and VEN were uniformly abraded, whereas the largest glass fillers in VED protruded from the surroundings and the prepolymer particles in VEP were deeper abraded than the bulk of the material. Toothbrushing with water only revealed not measurable wear. Roughness Ra was practically unchanged when compared with the preground samples before challenging with toothbrushes. Ranking by KHN was: MIF < VEN, VEP < VED, by DC: MIF < VEN < VEP < VED.

Conclusion: Filler concepts and monomer compositions affect wear and roughness of resin composites when tooth brushed with abrasive slurry. Toothbrushing without abrasive medium produced neither wear nor roughness. Careful polishing of resin composite restorations is an important determinant of wear and surface roughness.

How to cite this article: Suzuki T, Kyoizumi H, Araki Y, Finger WJ, Kanehira M. Toothbrush Abrasion of Resin Composites with Different Filler Concepts. World J Dent 2012;3(2):184-193.

Source of support: Nil

Conflict of interest: None declared

\section{INTRODUCTION}

New resin composite materials are frequently released to the dental marketplace. Manufacturers have a tendency to claim excellent performance of their products, but relevant information, especially from long-term clinical trials proving the claims are scarcely found. There are many crucial requirements to resin-based restoratives, both concerning biological, physical, and chemical characteristics that have to be fulfilled prior to marketing. Compliance with national and international standards is a minimum requirement. The ISO Technical Report No. 14569-1 (2007) gives some guidance on testing of wear by toothbrushing. ${ }^{1}$

Clinical studies on effects of toothbrushing are very seldom published. The reason might be the difficulty of standardization of the patients' daily life and oral hygiene habits that might have an impact on the degradation of restoration surfaces and longevity of restorations.

In contrast, there are a lot of commercialized or custommade brushing machines developed and described that might reasonably well simulate toothbrushing. ${ }^{2-9}$ However, there is no consensus, which machine might be most suitable. Although quantifiable evaluation parameters for the extent of wear and the morphology of the worn surfaces are available, some published articles are solely descriptive and thus less suitable for reasonable estimation and prediction of the effects of toothbrushing on resin composites. ${ }^{10,11}$

There is some confusion on toothbrush and toothpaste dentifrice effects on wear, and it is still a matter of dispute to which extent properties of the toothbrush contribute to surface wear of composites. ${ }^{12-15}$ Similarly, resistance of composite surfaces to different toothpastes is difficult to predict, since the composition of the individual pastes, in particular the kind of and loading with abrasive particles, may have different effects on different resin composites. ${ }^{16}$ Thus, it makes sense to use slurries of known abrasive particles as abrading media, rather than slurries of marketed toothpastes. $^{8}$

Modern resin composite materials differ much in terms of filler concepts and filler loading as well as in matrix monomer composition. Both filler grain size and distribution and polymerization quality of the resins are presumably of major importance for the resistance to toothbrushing of composite resins. Therefore, it is important to characterize the composite resin materials characteristics along with toothbrushing effects in order to evaluate possible relationships. ${ }^{17}$ Especially the degree of curing, that not only depends on the materials inherent properties but also largely 
on processing and operator effects, is believed to have some impact. $^{18-20}$

The aim of the present investigation was therefore to investigate effects of in vitro toothbrushing with and without a standardized abrasive slurry on wear and surface morphology of four resin composite materials with different filler concepts and matrix compositions. The null hypothesis tested is that composition and degree of polymerization of the resin composites have no effect on the amount of wear and on surface morphology caused by toothbrushing.

\section{MATERIALS AND METHODS}

The four resin composites used are shown in Table 1 together with their compositions and filler loading. According to the manufacturer MI FIL (MFI) contains Srdoped fillers with an average grain size of $200 \mathrm{~nm}$ and $16 \mathrm{~nm}$ silica fillers. Venus (VEN) is a conventional hybrid-type resin composite containing ground glass with an average grain size of $0.7 \mu \mathrm{m}$ and a minor amount of $\mathrm{SiO}_{2}$ microfiller $(0.04 \mu \mathrm{m})$. Venus Diamond (VED) and Venus Pearl (VEP) are nanohybrid resin composites that are very similar in matrix and filler composition. The main difference is the grain size distribution of the ground glass filler. In VED the largest particles are about $20 \mu \mathrm{m}$, in VEP only about $5 \mu \mathrm{m}$. The large glass fillers in VED are substituted in VEP with prepolymer particles. The percentage filler volume of VEP is $5 \%$ less. The matrix monomers used in MIF and VEN are conventional high-molecular Bis-GMA, BisMEPP or UDMA, mixed with TEGDMA as diluent monomer, whereas VED and VEP contain the new crosslinker monomer TCD-Di-HEA and UDMA.

\section{Specimen Preparation for Wear and Roughness Testing}

Rectangular Teflon molds $(12 \times 3 \times 3 \mathrm{~mm})$ were placed on Mylar strip covered glass slides, bulk filled with slight resin composite excess, covered with another Mylar strip and pressed flush with a top glass slide. The specimens were light activated only from one surface for 60 seconds with Translux Power Blue (LED Light, output: $650 \mathrm{~mW} / \mathrm{cm}^{2}$, Heraeus Kulzer GmbH, Hanau, Germany) in a slow scanning mode with the light-emitting window in contact with the strip-covered topside of the specimens. The polymerized beams were removed from the molds and immediately immersed in deionized water at room temperature for 24 hours before testing.

\section{Toothbrush Abrasion Testing}

Forty specimens were produced from each composite material and divided into four groups with 10 samples each

\begin{tabular}{|c|c|c|c|c|}
\hline $\begin{array}{l}\text { Material } \\
\text { (code) }\end{array}$ & $\begin{array}{l}\text { Batch/ } \\
\text { expiry }\end{array}$ & Manufacturer & Composition & $\begin{array}{l}\text { Filler content } \\
w t \% / v o l \%\end{array}$ \\
\hline $\begin{array}{l}\text { MI FIL } \\
\text { (MFI) }\end{array}$ & $\begin{array}{l}1101181 / \\
2014-01\end{array}$ & $\begin{array}{l}\text { GC Corporation, } \\
\text { Tokyo, Japan }\end{array}$ & $\begin{array}{l}\text { Matrix: UDMA, Bis-MEPP, } \\
\text { TEGDMA } \\
\text { Filler: Silica-nanofiller }(16 \mathrm{~nm}) \text {, } \\
\text { Sr-doped nanofiller }(200 \mathrm{~nm})\end{array}$ & $69 / 50$ \\
\hline $\begin{array}{l}\text { Venus } \\
\text { (VEN) }\end{array}$ & $\begin{array}{l}010402 / \\
2014-11\end{array}$ & $\begin{array}{l}\text { Heraeus Kulzer, } \\
\text { Hanau, Germany }\end{array}$ & $\begin{array}{l}\text { Matrix: Bis-GMA, TEGDMA } \\
\text { Filler: Ba-Al-F-glass } \\
\text { (average grain size: } 0.7 \mu \mathrm{m} \text {; max. }<2 \mu \mathrm{m} \text { ), } \\
\text { dispersed } \mathrm{SiO}_{2} \text { : } \\
\text { (average grain size: } 0.04 \mu \mathrm{m} \text { ) }\end{array}$ & $77 / 61$ \\
\hline $\begin{array}{l}\text { Venus Diamond } \\
\text { (VED) }\end{array}$ & $\begin{array}{l}010042 / \\
2014-12\end{array}$ & $\begin{array}{l}\text { Heraeus Kulzer, } \\
\text { Hanau, Germany }\end{array}$ & $\begin{array}{l}\text { Matrix: TCD-DI-HEA, } \\
\text { UDMA } \\
\text { Filler: Ba-Al-F-glass, } \\
\mathrm{SiO}_{2} \text { nanofiller } \\
\text { (grain size: } 5 \mathrm{~nm} \text { to } 20 \mu \mathrm{m} \text { ) }\end{array}$ & $81 / 64$ \\
\hline $\begin{array}{l}\text { Venus Pearl } \\
\text { (VEP) }\end{array}$ & $\begin{array}{l}\text { VP301110/ } \\
2014-05\end{array}$ & $\begin{array}{l}\text { Heraeus Kulzer, } \\
\text { Hanau, Germany }\end{array}$ & $\begin{array}{l}\text { Matrix: TCD-DI-HEA, } \\
\text { UDMA } \\
\text { Filler: Ba-Al-F-glass, } \\
\text { prepolymerized filler, } \\
\mathrm{SiO}_{2} \text { nanofiller } \\
\text { (grain size: } 5 \mathrm{~nm} \text { to } 5 \mu \mathrm{m} \text { ) }\end{array}$ & $\begin{array}{c}76 / 59^{*} \\
\text { (*58\% inorganic) }\end{array}$ \\
\hline
\end{tabular}

Bis-GMA: Bisphenol A diglycidyl ether methacrylate;

UDMA: 7,7,9-trimethyl-4,13-dioxo-3,14-dioxa-5,12-diazahexadecane-1,16-diyl-bis-methacrylate;

TEGDMA: 3,6-dioxaoctamethylendimethacrylate;

Bis-MEPP: 2,2-bis (4-methacryloxy polyethoxy phenyl) propane;

TCD-DI-HEA: Bis-(acryloyloxymethyl) tricyclo $(5.2 .1 .02,6)$ decane 
for allocation to toothbrush and toothbrush-slurry abrasion groups after two different surface finishing treatments. Ten specimens of each composite were mounted and glued with the light-activated side up on an acrylic resin plate $(48 \times 10$ $\times 3 \mathrm{~mm}$ ) in a row and in close contact to each other. Next to the resin beams three acrylic samples of same dimensions were mounted and glued at each end of the mounting plate. The exposed surfaces of two of the assembled specimen set-ups were ground on wet SiC paper, grit \#600 for 60 seconds, the two other assembled specimens were consecutively ground on SiC papers, grits \#2400 and \#4000 for 60 seconds.

For brushing with and without abrasive slurry a multistation brushing device (Tokyo Giken Inc, Tokyo, Japan) equipped with four lines of reciprocating Prospec Slim (GC Corp Tokyo, Japan) toothbrush heads (23 $\mathrm{mm}$ in length, $8 \mathrm{~mm}$ in width, medium hardness, $9.5 \mathrm{~mm}$ filament length) was used. Each plate holder with 10 composite specimens was covered with a $1 \mathrm{~mm}$ wide metal shield at each side of the $10 \mathrm{~mm}$ long specimens prior to mounting into the testing machine underneath a clamped toothbrush head.

Instead of toothpaste aqueous slurry of $150 \mathrm{~g}$ of calcium carbonate (Calcium carbonate 030-00385, Wako Pure Chemical Industries. Ltd., Osaka, Japan) dispensed in $100 \mathrm{ml}$ of water was used as the abrasive medium. The calcium carbonate powder has a purity of $99.5 \%$ (mass/mass), and the average particle size is $5.2 \mu \mathrm{m}$. For the toothbrush test deionized water was used instead of abrasive slurry.

The specimens were immersed in the abrasive slurry or water during the five times repeated 10,000 forth-and-back brushing strokes $(60 \mathrm{~Hz})$ in a direction perpendicular to the lengths of the composite beams. The static load on the toothbrush heads was $5 \mathrm{~N}$. The specimens were exposed to a total of 50,000 brushing cycles. After each 10,000 strokes the slurry was changed. Prior to each test run new toothbrush heads were mounted. The test was performed at ambient laboratory atmosphere $\left(23^{\circ} \mathrm{C}\right.$ and $50 \pm 15 \%$ relative humidity).

\section{Measurement of Depth of Wear and Determination of Surface Roughness}

The baseline surface roughness of each of the mounted specimens was determined with a profilometer (Surfcom 480A, Tokyo Seimitsu Co., LTD, Tokyo, Japan) equipped with a diamond pick-up (tip radius: $5 \mu \mathrm{m}$, load: $4 \mathrm{mN}$ ).

After each 10k brushing cycles the specimens were thoroughly rinsed with water and dried with a short air blast before mounting on the stage of the surface analyzer for tracing. For determination of the depth of wear of each composite beam the stylus traversed centrally $11 \mathrm{~mm}$ from one shielded reference plane of the specimen to the opposite one. The depth was measured graphically from the registered stylus trace profile as the largest difference in $\mu \mathrm{m}$ between a line connecting the opposing reference planes and the deepest portion on the trace.

Surface roughness $(\mathrm{Ra})$ was determined at each measuring stage in the central portion of the composite beam, close to the centerline $(1.25 \mathrm{~mm}$ trace length, $0.6 \mathrm{~mm} / \mathrm{s}$, cut-off $0.25 \mathrm{~mm}$ ).

ANOVA and post-hoc tests at a significance level $\mathrm{p} \leq 0.05$ were used to analyze statistically the depth of wear and roughness data. Wear data were additionally evaluated by linear regression analyzes.

\section{Knoop Hardness Measurements}

Three cylindrical specimens ( $5 \mathrm{~mm}$ in diameter, $3 \mathrm{~mm}$ thick) of each composite resin material were produced in a steel mold lined with Mylar sheets. The samples were light activated from one side only with Translux Power Blue in contact with the Mylar strip covered surface for 20 seconds. The activated top surface was polished on wet $\mathrm{SiC}$ paper, grit \#4000 for 60 seconds. After 24 hours immersion in $23^{\circ} \mathrm{C}$ water Knoop hardness numbers (KHN: $\mathrm{kgf} / \mathrm{mm}^{2}$ ) were determined from indentations at five locations close to the center of the specimen (Hardness Tester HM-102, Akashi Co.,Yokohama, Japan; $0.25 \mathrm{~N}$ load; 15s). Data analysis was done by ANOVA and Tukey's post-hoc test $(\mathrm{p} \leq 0.05)$.

\section{Degree of Conversion of Double Bonds (DC)}

For determination of the DC, specimens were prepared as described for KHN testing above. Spectra of the uncured and cured materials were measured by a Raman spectrophotometer NRS-3000FL (JASCO Corporation, Tokyo, Japan) fitted with a solid-state diode laser (532 nm, $1.2 \mathrm{~mW}$ ). The spectral resolution was approximately $1 \mathrm{~cm}^{-1}$. Through the microscope objective (UMPLFL20x, NA $=0.46$, Olympus) the spatial resolution was approximately $3 \mu \mathrm{m}$. Raman spectra were obtained in the region 1970-975 $\mathrm{cm}^{-1}$. Eight accumulations throughout 5 seconds measurement were performed.

For MIF and VEN the degree of conversion (DC) is determined as the percentage of vinyl functions converted to aliphatic functions comparing the vibration band of the residual unpolymerized (meth) acrylate $\mathrm{C}=\mathrm{C}$ bond at $1640 \mathrm{~cm}^{-1}$ against the aromatic $\mathrm{C}=\mathrm{C}$ stretching band at $1610 \mathrm{~cm}^{-1}$ used as internal standard. The DC is calculated as follows: $\mathrm{DC}(\%)=100\left[1-\left(\mathrm{R}_{\text {polymerized }} / \mathrm{R}_{\text {unpolymerized }}\right)\right]$, where $\mathrm{R}$ is the ratio between the peak area at $1640 \mathrm{~cm}^{-1}$ / peak area at $1610 \mathrm{~cm}^{-1}$.

VED and VEP show no aromatic stretching band at $1610 \mathrm{~cm}^{-1}$, therefore the band at $1600 \mathrm{~cm}^{-1}$, the $\mathrm{C}=\mathrm{C}$ 
stretching mode in the acryloyloxymethyl group was used as internal standard.

On each specimen percentage DC was determined at the central location of the disk.

\section{Scanning Electron Microscopy}

One random sample of each material, representing pretreatment and brushing group was selected after 50,000 brushing cycles for SEM examination (Type VE-8800, Keyence Inc., Osaka, Japan). Specimens were sputter-coated with platinum and photographs were taken of representative areas at 3000-fold magnification $(10 \mathrm{kV})$.

\section{RESULTS}

Figures 1 and 2 show the means of the maximum wear depths of the tested materials by numbers of brushing cycles with toothpaste slurry. Figure 1 gives the wear data of composites after pregrinding on $\mathrm{SiC}$ paper \#600, whereas Figure 2 shows the results obtained from specimens preground on $\mathrm{SiC}$ paper \#4000. The linear regression lines describe the best fit of the mean wear depths according to the least square distance method. All regression lines were set through zero, based on the obvious condition that no brushing produces no wear. All coefficients of determination $\mathrm{r}^{2}$ for the different products and pregrinding conditions are $\geq 0.94$. Apart from VED, wear of the composites was significantly higher on specimens preground with the relatively coarse \#600 paper.

When wear was determined as a result of toothbrushing in water no loss of substance for any of the tested materials was measurable.

The bar diagrams in Figures 3 and 4 illustrate the means and standard deviations of the specimens' surface roughness (Ra) by number of brushing cycles with abrasive slurries for specimens preground on SiC papers \#600 and \#4000 respectively. The bar clusters represent the four resin composites. For each material's relationship between Ra and number of cycles one-way ANOVA was calculated. Same lower-case letters within a material group indicate that surface roughness is not significantly different. For specimens preground on $\mathrm{SiC} \# 600$ significant differences by materials were found: MIF,VEN $<$ VEP $<$ VED. When specimens were preground on $\mathrm{SiC}$ paper \#4000 the following homogeneous groups were identified: MIF, VEN $<$ VEN, VEP $<$ VED.

SEM microphotographs (Fig. 5) from representative composite surfaces (preground on $\mathrm{SiC} \mathrm{\# 600)} \mathrm{after} \mathrm{50,000}$ brush strokes with abrasive slurry show quite uniformly abraded MIF and VEN surfaces with few pit holes left after filler exfoliation, whereas the surface of VED is

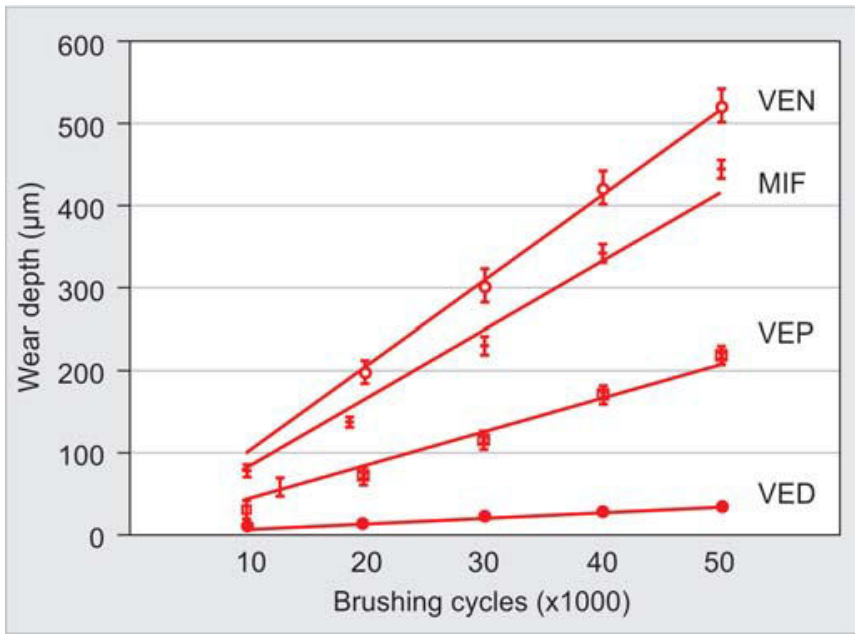

Fig. 1: Depth of wear $(\mu \mathrm{m})$ after toothbrushing of resin composites with abrasive slurry by brushing cycles. Composite specimens were preground on SiC paper \#600. Linear regression lines set through zero. Coefficients of determination $r^{2} \geq 0.94(n=10)$

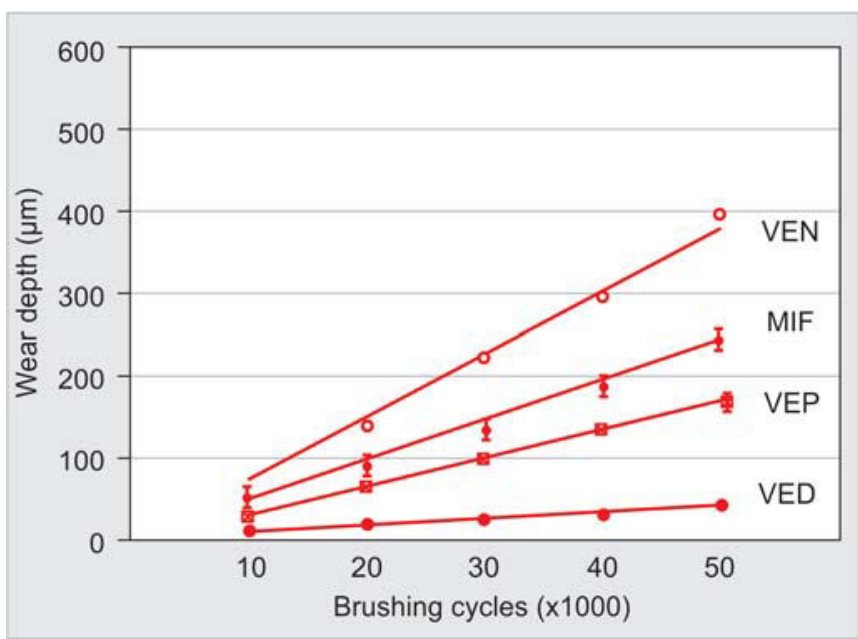

Fig. 2: Depth of wear $(\mu \mathrm{m})$ after toothbrushing of resin composites with abrasive slurry by brushing cycles. Composite specimens were preground on SiC paper \#4000. Linear regression lines set through zero. Coefficients of determination $r^{2} \geq 0.98(n=10)$

characterized by highly protruding large filler particles in a rather uniformly worn surrounding area containing much smaller filler particles. With VEP large prepolymer fillers are seen underneath the level of the surrounding glass filler rich composite area. Almost identical surface morphology and abrasion features are shown in Figure 6 representing samples of the four materials, preground on $\mathrm{SiC} \mathrm{\# 4000} \mathrm{after}$ 50,000 brushing cycles in slurry.

Brushing of specimens in water (Figs 7 and 8), when preground on $\mathrm{SiC} \# 600$ or \#4000, had no effect on the surface roughness irrespective of the numbers of strokes. Significant differences in Ra by material when preground on SiC \#600 were VEN,VEP $<$ VEN, VED $<$ MIF, when preground on $\mathrm{SiC} \# 4000$ the following Tukey ranking was 


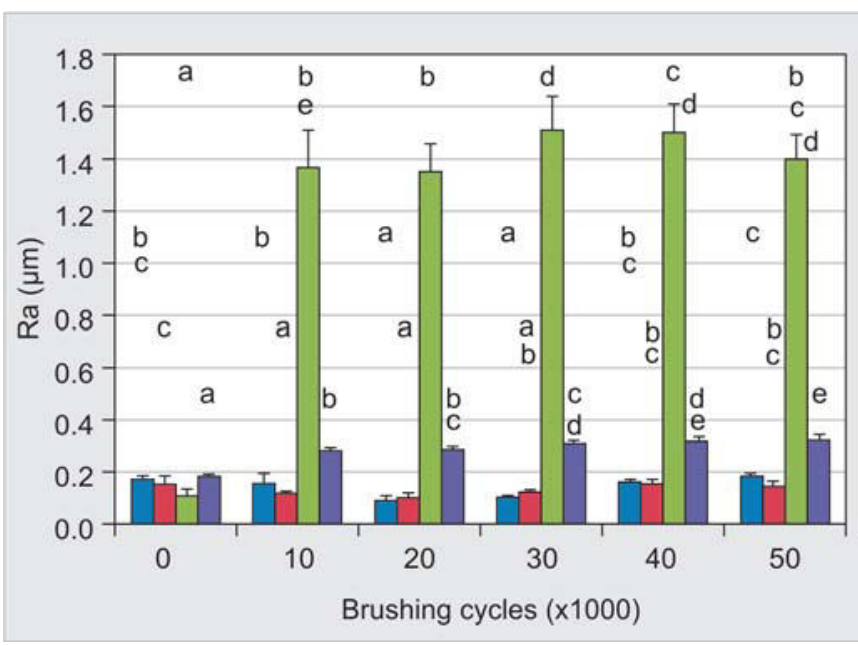

Fig. 3: Surface roughness $\mathrm{Ra}(\mu \mathrm{m})$ after toothbrushing of resin composites with slurry by brushing cycles. Composite specimens were preground on SiC paper \#600 $(n=10)$. In each cluster, the bars from left to right represent MIF, VEN, VED and VEP. Same lower-case letters over bars of each individual material denote groups that are not significantly different $(p \leq 0.05)$
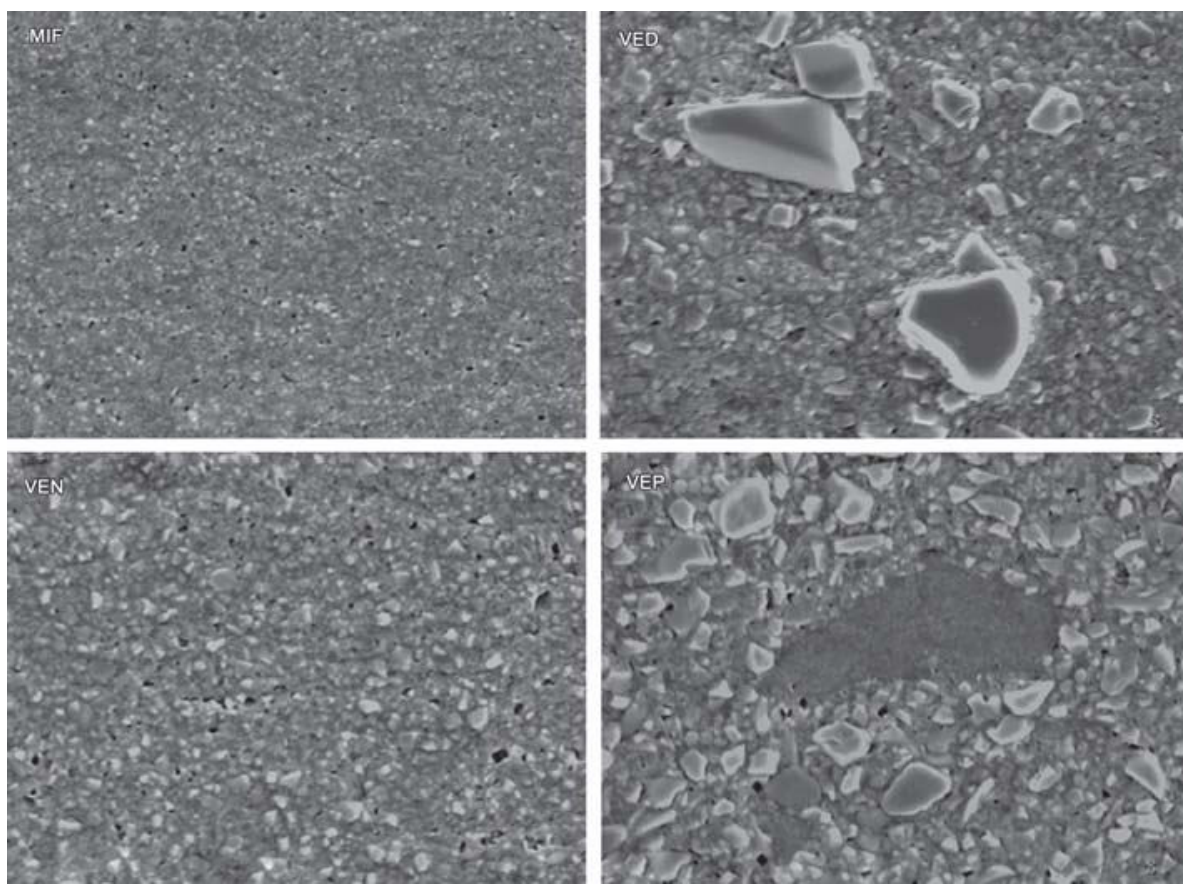

Fig. 5: SEM microphotographs (3000x) of representative resin composite specimens preground on SiC paper \#600 after 50,000 brushing cycles with abrasive slurry. MIF and VEN show uniformly abraded surfaces with some pitting. Large glass fillers protruding from filler-rich polymer surrounding characterize VED. The prepolymer fillers in VEP are deeper abraded than the surrounding filled polymer. Pits denote particle exfoliation

Table 2: KHN and DC (\%) of resin composites tested $(n=3)$

\begin{tabular}{llll}
\hline Material & $\begin{array}{l}\text { Filler content } \\
\text { wt\%/vol\% }\end{array}$ & KHN & DC (\%) \\
\hline MIF & $69 / 50$ & $47.7 \pm 5.4^{\mathrm{a}}$ & 59.3 \\
VEN & $77 / 61$ & $69.8 \pm 5.7^{\mathrm{b}}$ & 74.4 \\
VED & $81 / 64$ & $77.9 \pm 7.9^{\mathrm{c}}$ & 80.8 \\
VEP & $76 / 59$ & $67.4 \pm 4.1^{\mathrm{d}}$ & 80.6 \\
\hline Significance & & $\mathrm{p}<0.001$ & \\
\hline
\end{tabular}

Same lower-case letters denote no significant difference in $\mathrm{KHN}(\mathrm{p} \leq 0.05)$ 

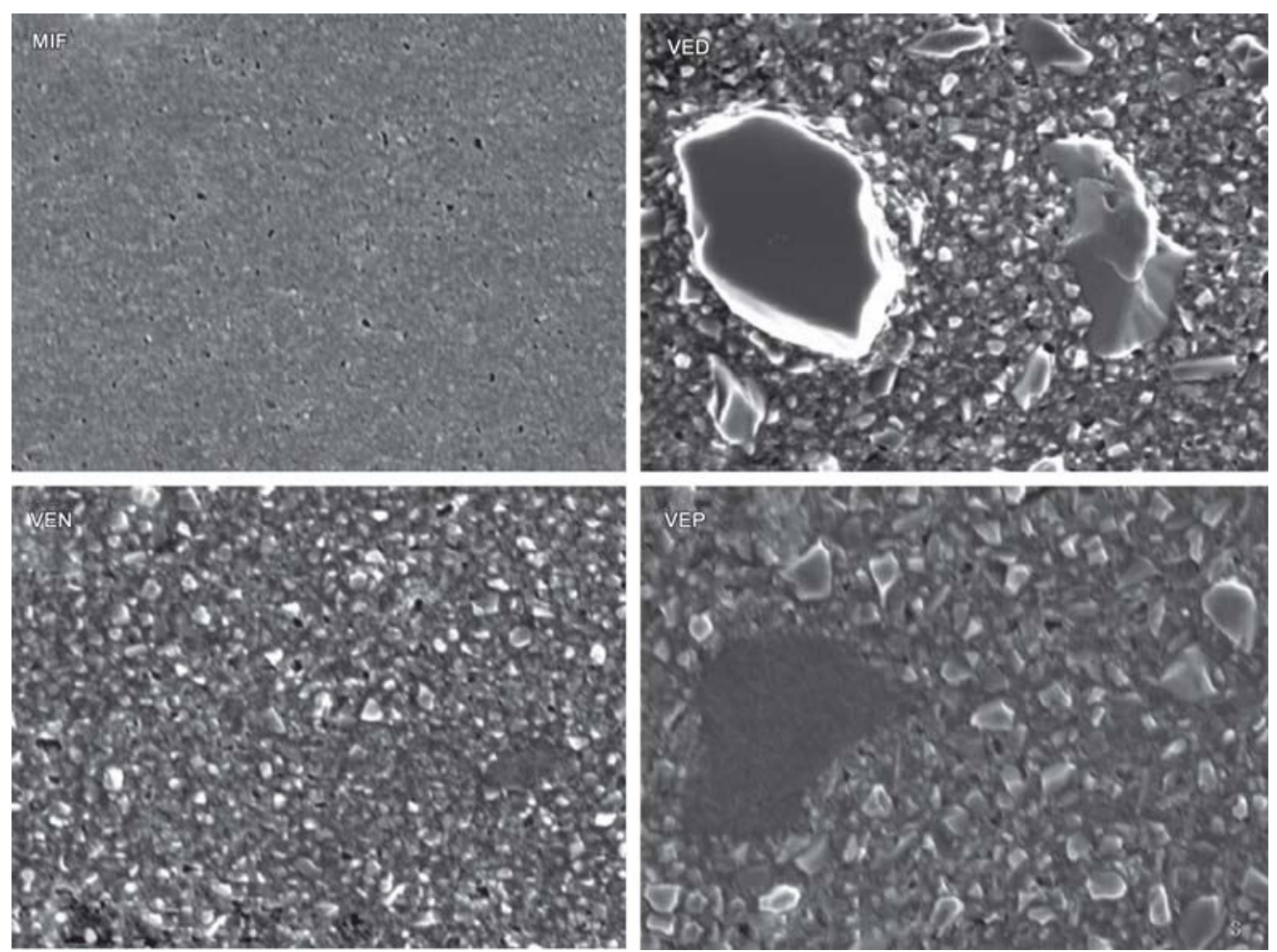

Fig. 6: SEM microphotographs (3.000x) of representative resin composite specimens preground on SiC paper \#4000 after 50,000 brushing cycles with abrasive slurry. Same features as in Figure 5

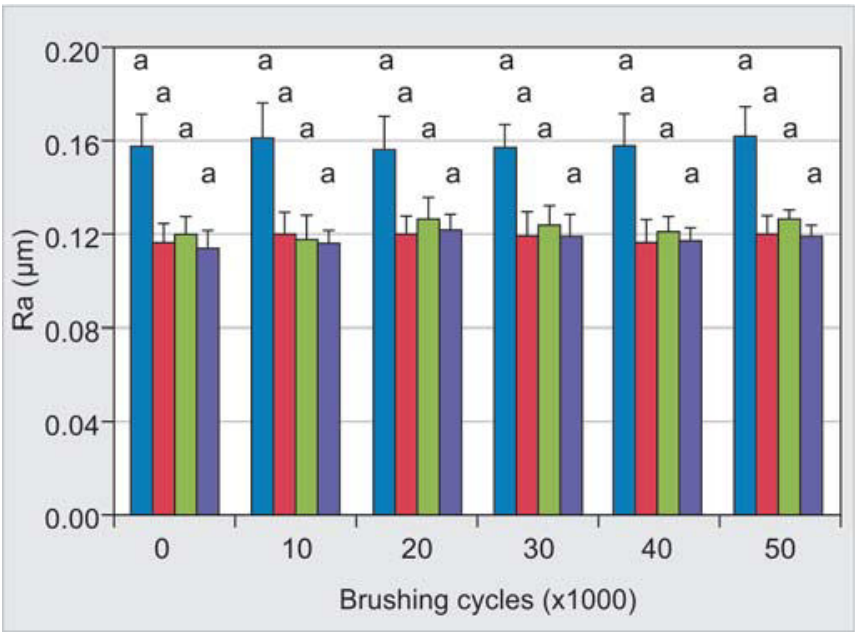

Fig. 7: Surface roughness $\mathrm{Ra}(\mu \mathrm{m})$ after toothbrushing with water of resin composites by brushing cycles. Composite specimens were preground on SiC paper \#600 $(n=10)$. In clusters, the bars from left to right represent MIF, VEN, VED and VEP. Same lower-case letters over bars of each individual material denote groups that are not significantly different $(p \leq 0.05)$

obtained: MIF $<$ VEN $<$ VEP $<$ VED. The SEMs shown in Figures 9 and 10 from samples after 50,000 brush strokes in water, display typical surfaces of specimens after pregrinding and prior to brushing. For samples of MIF and VEN even scratches produced during pregrinding are still visible.

Table 2 shows the mean Knoop hardness figures and the degrees of conversion of double bonds for the materials

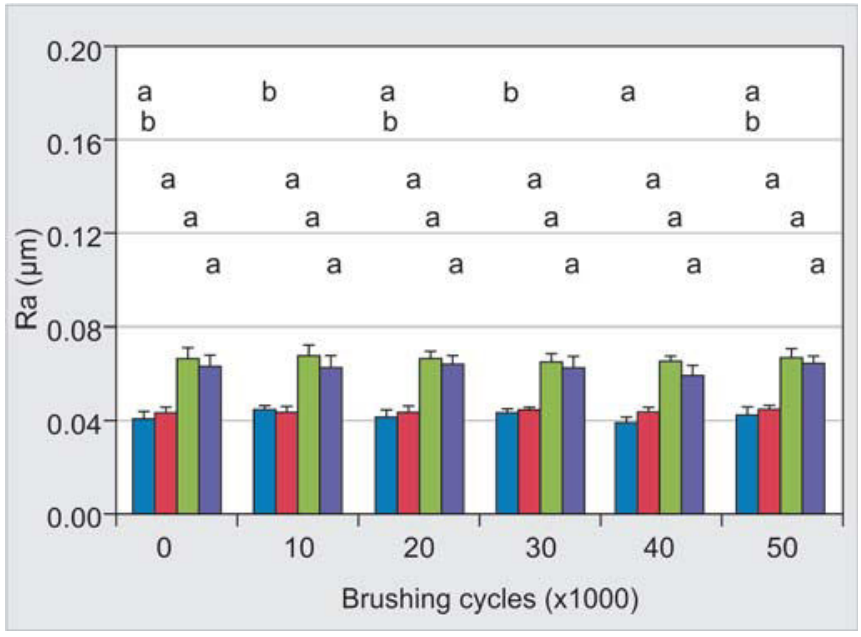

Fig. 8: Surface roughness $\mathrm{Ra}(\mu \mathrm{m})$ after toothbrushing with water of resin composites by brushing cycles. Composite specimens were preground on SiC paper \#4000 $(n=10)$. In clusters, the bars from left to right represent MIF, VEN, VED and VEP. Same lower-case letters over bars of each individual material denote groups that are not significantly different $(p \leq 0.05)$

tested. The KHN of the comparatively low filler-loaded MIF is significantly smaller than that of VEN and VEP. The highest KHN is recorded for VED. The percentage DC values calculated from Raman spectra were: MIF (59.3\%) $<\operatorname{VEN}(74.4 \%)<\operatorname{VEP}(80.6 \%)<\operatorname{VED}(80.8 \%)$.

\section{DISCUSSION}

The null hypothesis tested in this study that composition and degree of polymerization of the resin composites have 

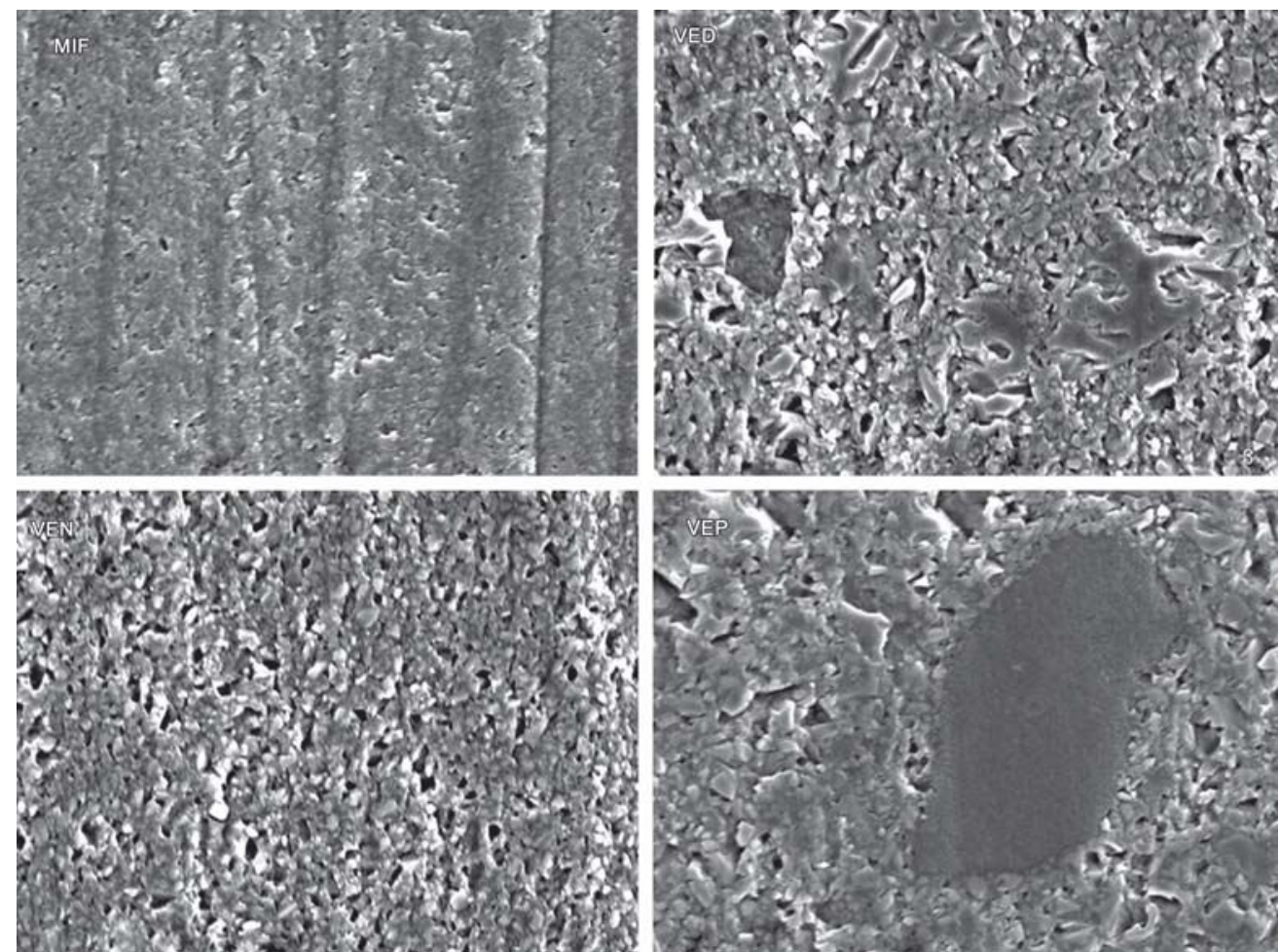

Fig. 9: SEM microphotographs (3000x) of representative resin composite specimens preground on SiC paper \#600 after 50,000 brushing cycles with water. For MIF and VEN scratches produced at pregrinding are visible. No signs of wear are detected
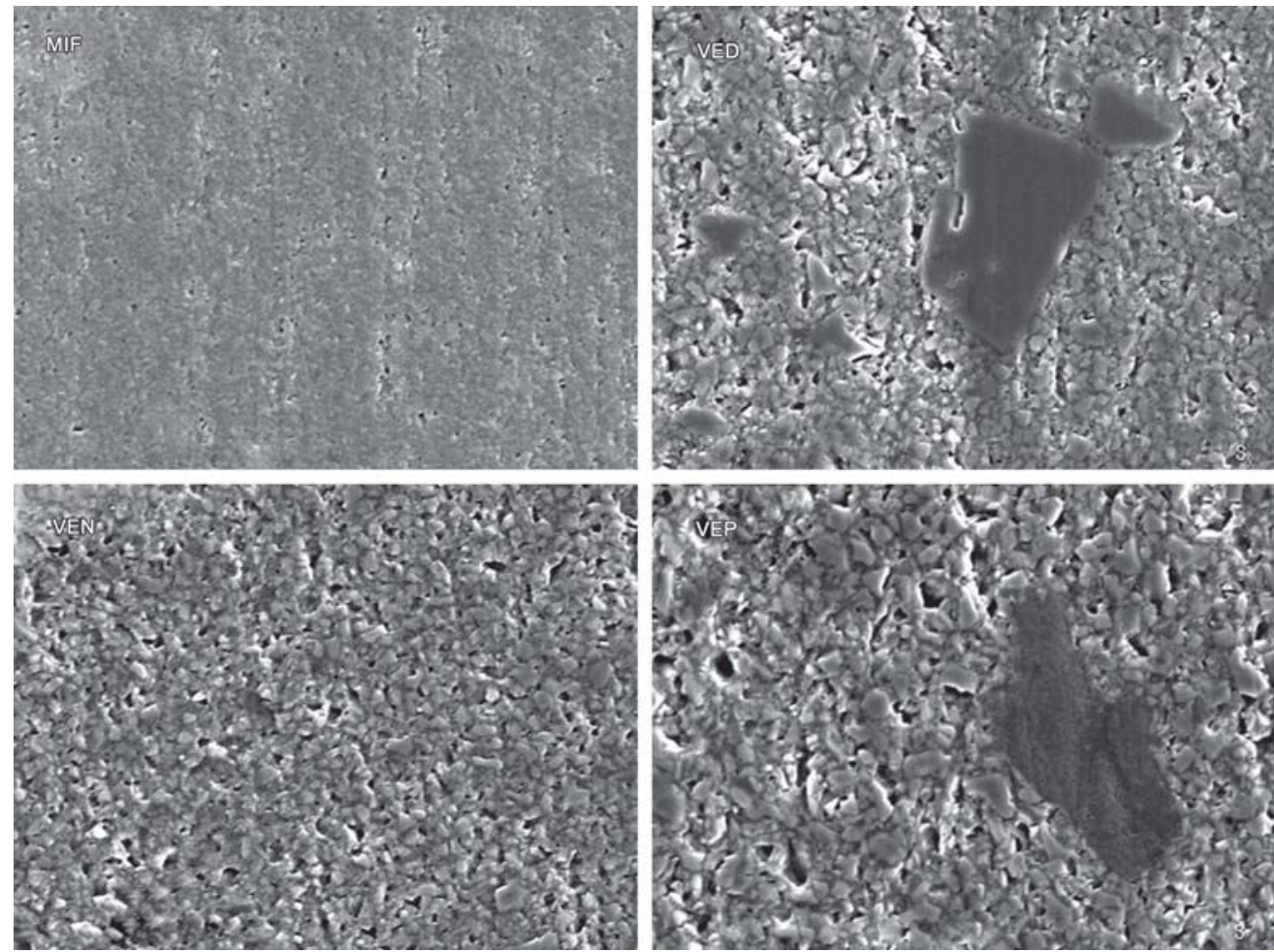

Fig. 10: SEM microphotographs (3000x) of representative resin composite specimens preground on SiC paper \#4000 after 50,000 brushing cycles with water. No signs of wear are detected

no effect on the amount of wear and on surface morphology caused by toothbrushing has to be partly rejected. Toothbrushing with the abrasive slurry resulted in significantly different amounts of wear among the materials investigated, whereas toothbrushing with water only produced no measurable wear for any of the resin composite materials. Regarding surface roughness as a result of the three-body wear testing also significantly different surface textures of the individual materials were revealed.

The calcium carbonate abrasive powder used as 'toothpaste' slurry had a Mohs hardness number 3 and an average particle grain size of $5.2 \mu \mathrm{m} .{ }^{8}$ Intentionally, we 
used this standardized slurry instead of diluted toothpaste in order to prevent possible effects of unknown components of the composition. Moreover, calcium carbonate is the abrasive used in many commercial toothpastes recommended for daily use due to the comparatively mild abrasivity. ${ }^{7}$

For our trial we selected arbitrarily a medium hard type of toothbrushes that is frequently sold in Japan. ${ }^{13}$ It is well documented that the type of toothbrush used has no effect on abrasion when used with water, however some effect on wear when carrying toothpaste. ${ }^{10,14,15}$ Soft brushes cause reportedly more abrasion than medium or hard brushes, which is explained with increased retention of toothpaste by smaller diameter filaments and denser tufts on soft brushes and the greater flexion of filaments increasing the area of contact with the surface. ${ }^{13,15}$

In different in vitro trials largely differing loads on toothbrushes are applied. ${ }^{7}$ In this trial we selected to apply a brushing force of $5 \mathrm{~N}$ which is twice the maximum force recommended in ISO/TR. ${ }^{1}$ The increased load was chosen anticipating that higher force will result in higher wear and roughness. ${ }^{7,21}$ Especially, with highly wear resistant resin composites higher load of the abrasive slurry will produce measurable wear depths even at smaller numbers of brush strokes. Van der Weiden et $\mathrm{al}^{22}$ determined the toothbrushing force of 94 people. The average force reported was $3.3 \mathrm{~N}$, with a wide spreading from 1.4 to $7.2 \mathrm{~N}$. Similar brushing forces were found by other authors. ${ }^{23,24}$ On the basis of these reports and in agreement with Cho et $\mathrm{al}^{25}$ and Senawongse and Pongprueksa ${ }^{6}$ it was justifiable to use $5 \mathrm{~N}$ brushing load.

It is important to use many toothbrushing cycles for the evaluation of wear and roughness, because a large number of strokes is expected to produce unequivocal wear results even on highly wear-resistant materials. Additionally, large numbers of brushing cycles, interrupted for wear and surface roughness registrations at different stages, can reveal linearity or nonlinearity of brushing effects overtime. According to several reports 10,000 brushing cycles reflect approximately 1 year of toothbrushing. ${ }^{26,27}$ Therefore, our data simulate in total 5 years of toothbrushing.

Several methods for quantification of wear have been suggested. Measuring of the thickness of abraded specimens using calipers ${ }^{5}$ and determination of weight loss after challenging ${ }^{1,27}$ are simple but rather rough measures. More common is graphical determination of the maximum depth of wear from profilometer traces. ${ }^{8}$ New sophisticated measuring methods, such as $3 \mathrm{D}-\mathrm{CCD}$ microscopy ${ }^{28}$ and confocal laser microscopy ${ }^{20}$ have a potential to record even minor amounts of wear already after comparatively few brushing cycles. The sensitivity of the profilometer method used in this trial is however, high enough for sufficient discrimination among testing stages and materials.

The present study revealed that the roughness of specimens before toothbrush-slurry challenging was an important determinant of wear and surface roughness. This is why ISO/TR 14569-1 requests wet-grinding of the specimens with grade $1000 \mathrm{SiC}$ paper prior to brushing. ${ }^{1}$ Our results confirm the importance of good polishing as a preventive measure to keep the effect of toothpaste abrasion low. Concerning roughness resulting from toothbrushing with abrasive slurry no practically significant effects were seen at the different measuring stages, which confirms that roughness is rather an inherent material characteristic, mainly related to filler loading and characteristics.

As shown in this trial it is mandatory to take a look at both quantitative and qualitative aspects of abrasivity. ${ }^{15,16}$ Determination of single parameters only, such as wear or roughness, make it very difficult to analyze the mechanisms behind the effects. Therefore additionally visualization methods, such as SEM should be applied.

The results acquired with MIF are interesting. Due to the comparatively low degree of filler loading (50 vol\%) the matrix polymer is prone to wear by scratching with the abrasive particles and subsequent loss of substance, including the very fine average filler of $200 \mathrm{~nm}$. The low $\mathrm{KHN}$ of MIF is also an indicator for low filler loading. The small surface roughness produced after brushing with water or abrasive slurry is an expression of the fine nanofiller particles. SEMs demonstrate a very uniform abrasion pattern with relatively few pit holes. The DC was rather high with almost $60 \%$. It has to be kept in mind that KHN and DC measurements reflect different changes in the polymer during and after curing. KHN responds mainly to the crosslinking density of the polymer, whereas DC, measuring the amount of conversion of carbon double bonds to single bonds, summarizes several events, such as chain elongation reaction with initiator radicals and crosslinking.

VEN is a traditional hybrid-type resin composite with 61 vol\% loading of ground glass particles at average grain size of $0.7 \mu \mathrm{m}$ plus an addition of a few percent microfiller. The SEMs demonstrate rather uniformly worn surfaces. However, due to the larger filler dimensions compared to MIF exfoliation under action of the abrasive leaves deeper defects and consecutively higher wear. In spite of the KHN of almost 70 and the high DC of $74.4 \%$, toothbrush-slurry wear was high indicating that filler grain size and or fillermatrix coupling might be the determinants of the relatively high wear.

VED and VEP contain both the same monomers. The DC of more than $80 \%$ found for both products was 
remarkably high. Similar high values for the degree of conversion of Venus Diamond were reported by Cerutti et $\mathrm{al}^{29}$ and Boaro et $\mathrm{al}^{30}$ using Raman and FTIR spectroscopy, respectively. The main crosslinker included in VED is TCD-DI-HEA, a high molecular mass urethane diacrylate. It may be speculated that VED and VEP cure comparatively slowly, leading to many double bond conversions by chain elongation and crosslinking before finally, due to immobilization of the system, no further reaction between double bonds and remaining radicals is possible. ${ }^{31}$ The surface hardness of VED is high, presumably as a result of high crosslinking density together with the high filler load. The KHN measured for VEP is in accordance with the 5\% lower degree of filler volume smaller than the one for VED. Since, VED and VEP are very similar products it is interesting to hypothesize about the reasons for the highly different wear results obtained with the abrasive slurry. In VED the largest glass fillers are about $20 \mu \mathrm{m}$. During the wear process the filler-rich polymer phase around these large glass particles is gradually removed, while the huge particles protruding from the surface prevent fast and deep abrasion of the surroundings. As a result, wear is very limited, whereas surface roughness is considerably higher than with composites like MIF and VEN. Generally, composites containing smaller average filler particles show less increase in roughness than ones with larger fillers. ${ }^{32}$

With the introduction of VEP the manufacturer has modified the filler concept presented in VED. The volume percentage of filler is reduced and the largest fillers in VEP are $5 \mu \mathrm{m}$ only. The huge glass particles seen in VED were substituted with prepolymer fillers, whereas the matrix remained unchanged. This product modification seems to be a good compromise when compared with VED. Wear under the abrasive slurry is increased, however still significantly smaller than with MIF and VEN, and surface roughness is significantly decreased compared with VED. The SEM photographs reveal the reasons. During 'toothpaste' wear the exposed prepolymer fillers are preferentially abraded, exposing the more wear resistant glass filler-loaded material to the action of the abrasive.

In summary, filler concepts and monomer compositions affect wear and roughness of resin composites when toothbrushed with abrasive slurry. Toothbrushing without abrasive medium produced neither wear nor roughness. Careful polishing of resin composite restorations is an important determinant of wear and surface roughness. Smoothly polished surfaces of resin composite restorations are mandatory, since roughness figures exceeding a threshold $\mathrm{Ra}$ value of $0.2 \mu \mathrm{m}$ have significant effects on in vivo plaque accumulation. ${ }^{33,34} \mathrm{~A}$ clinical study is in progress to investigate whether the present in vitro data, acquired underhigh brushing load are indicative to the wear effects resulting from patients, daily tooth brushing.

\section{ACKNOWLEDGMENT}

The authors gratefully acknowledge donation of the resin composite materials by GC Corporation and Heraeus Kulzer. This study was in part supported by a grant of the Lowcarbon Society at Tohoku University in Sendai, Japan.

\section{REFERENCES}

1. ISO TR. Dental materials guidance on testing of wear: Part 1: Wear by tooth brushing. Technical Report 2007;14569:1.

2. Heath JR, Wilson HJ. Abrasion of restorative materials by toothpaste. J Oral Rehabil 1976;3:121-38.

3. Turssi CP, de Magalhães CS, Serra MC, Rodrigues AL. Surface roughness assessment of resin-based materials during brushing preceded by pH-cycling simulations. Oper Dent 2001;26: 576-84.

4. Heintze SD, Forjanic M. Surface roughness of different dental materials before and after simulated toothbrushing in vitro. Oper Dent 2005;30:617-26.

5. Texeira ECN, Thompson JL, Piascik JR, Thompson JY. In vitro toothbrush-dentifrice abrasion of two restorative composites. J Esthet Restor Dent 2005;17:172-82.

6. Senawongse P, Pongprueksa P. Surface roughness of nanofill and nanohybrid resin composites after polishing and brushing. J Esthet Restor Dent 2007;19:265-75.

7. Parry J, Harrington E, Rees GD, McNab R, Smith AJ. Control of brushing variables for the in vitro assessment of toothpaste abrasivity using a novel laboratory model. J Dent 2008;36: 117-24.

8. Suzuki T, Kyoizumi H, Finger WJ, Kanehira M, Endo T, Utterodt A, Hisamitsu H, Komatsu M. Resistance of nanofill and nanohybrid resin composites to toothbrush abrasion with calcium carbonate slurry. Dent Mater J 2009;28:708-16.

9. Jain V, Platt JA, Moore BK, Borges GA. In vitro wear of new indirect resin composites. Oper Dent 2009;34:423-28.

10. Van Dijken JWV, Stadigh J, Meurman JM. Appearance of finished and unfinished composite surfaces after toothbrushing. Acta Odontol Scand 1983;41:377-83

11. Moraes RR, Gonçalves L, Lancellotti AC, Consani S, CorrerSobrinho L, Sinhoreti MA. Nanohybrid resin composites: Nanofiller loaded materials or traditional microhybrid resins. Oper Dent 2009;34:551-57.

12. Rawls HR, Mkwayi-Tulloch NJ, Casella R, Cosgrove R. The measurement of toothbrush wear. J Dent Res 1989;68:1781-85.

13. Dyer D, Addy M, Newcombe RG. Studies in vitro of abrasion by different manual toothbrush heads and a standard toothpaste. J ClinPeriodontol 2000;27:99-103.

14. Wiegand A, Kuhn M, Sener B, Roos M, Attin T. Abrasion of eroded dentin caused by toothpaste slurries of different abrasivity and toothbrushes of different filament diameter. J Dent 2009;37:480-84.

15. Tellefsen G, Liljeborg A, Johannsen A, Johannsen G. The role of the toothbrush in the abrasion process. Int J Dent Hygiene 2011;9:284-90

16. Redmalm G, Rýden H. Dentifrice abrasivity. The use of laser light and supplemental techniques for characterizing toothpastes containing different abrasives. An in vitro study. Swed Dent J 1984;8:57-66. 
17. Kawai K, Iwami Y, Ebisu S. Effect of resin monomer composition on toothbrush wear resistance. J Oral Rehabil 1998;25:264-68.

18. Tanoue N, Matsumura H, Atsuta M. Analysis of composite type and different sources of polymerization light on in vitro toothbrush/dentifrice abrasion resistance. J Dent 2000;28: 355-59.

19. Martinelli J, Pires-de-Souza F, Casemiro LA, Tirapelli C, Panzeri $\mathrm{H}$. Abrasion resistance of composites polymerized by lightemitting diodes (LED) and halogen light-curing units. Braz Dent J 2006;17:29-33.

20. Murakami M. Surface properties of an indirect composite polymerized with five laboratory light polymerization systems. J Oral Sci 2009;51:215-21.

21. Lewis R, Dwyer-Joyce RS, Pickles MJ. Interaction between toothbrushes and toothpaste abrasive particles in simulated tooth cleaning. Wear 2004;257:368-76.

22. Van der Weiden GA, Timmerman MF, Danser MM, van der Velden U. Relationship between the plaque removal efficacy of a manual toothbrush and brushing force. J Clin Periodontol 1998;25:413-16.

23. Volpenhein DW, Walsh ME, Dellerman PA, Burkett TA. A new method for in vitro evaluation of the interproximal penetration of manual toothbrushes. J Clin Dent 1994;5:27-33.

24. Kon M, Kakuta K, Ogura H. Effects of occlusal and brushing forces on wear of composite resins. Dent Mater J 2006;25: 183-94.

25. Cho LR, Yi YJ, Heo SJ. Effect of tooth brushing and thermal cycling on a surface change of ceromers finished with different methods. J Oral Rehabil 2002;29:816-22.

26. Turssi CP, Hara AT, de Magalhães CS, Serra MC, Rodrigues AL Jr. Influence of storage regime prior to abrasion on surface topography of restorative materials. J Biomed Mater Res 2003;65:227-32.

27. Wang L, Garcia FC, Amarante de Araujo P, Franco EB, Mondelli RF. Wear resistance of packable resin composites after simulated toothbrushing test. J Esthet Restor Dent 2004;16:303-14.

28. Wonglamsam A, Kakuta K, Ogura H. Effects of occlusal and brushing cycles on wear of composite resins in combined wear test. Dent Mater J 2008;27:243-50.

29. Cerutti F, Acquaviva PA, Gagliani M, Madini L, Mangani F, Depero LE, Cerutti A. Relevance of different polymerisation methods on light-curing composites conversion degree. J Dent Res 2009;88(Sp Iss A):(Abstr 301)

30. Boaro L, Versluis A, Braga R. Degree of conversion and postgel shrinkage of low shrinkage composites. J Dent Res 2010;89 (Sp Iss B): (Abstr 319).
31. Marchesi G, Breschi L, Antoniolli F, Di Lenarda R, Ferracane J, Cadenaro M. Contraction stress of low-shrinkage composite materials assessed with different testing systems. Dent Mater 2010;26:947-53

32. Da Costa J, Adams-Beluska A, Riley K, Ferracane JL. The effect of various dentifrices on surface roughness and gloss of resin composites. J Dent 2010;38s:e123-28.

33. Bollen CML, Papaioannou W, van Eldere J, Schepers E, Quirynen M, van Steenberghe D. The influence of abutment surface roughness on plaque accumulation and peri-implant mucositis. Clin Oral Imp Res 1996;7:201-11.

34. Bollen CML, Lambrechts P, Quirynen M. Comparison of surface roughness of oral hard materials to the threshold surface roughness for bacterial plaque retention: A review of the literature. Dent Mater 1997;13:258-69.

\section{ABOUT THE AUTHORS}

\section{Toshimitsu Suzuki}

Associate Professor, Department of Clinical Cariology, Showa University, School of Dentistry, Tokyo, Japan

\section{Hideaki Kyoizumi}

Assistant Lecturer, Department of Clinical Cariology, Showa University, School of Dentistry, Tokyo, Japan

\section{Yasuyuki Araki}

Assistant Professor, Institute of Multidisciplinary Research for Advanced Materials, Tohoku University, Sendai, Japan

\section{Werner J Finger}

Guest Professor, Department of Restorative Dentistry, Division of Operative Dentistry, Tohoku University, Graduate School of Dentistry Sendai, Japan

\section{Masafumi Kanehira}

Assistant Professor, Department of Restorative Dentistry, Division of Operative Dentistry, Tohoku University, Graduate School of Dentistry, Sendai, Japan

\section{CORRESPONDING AUTHOR}

Toshimitsu Suzuki, Associate Professor, Department of Clinical Cariology, Showa University, School of Dentistry, 2-1-1 Kitasenzoku, Ohta-ku, Tokyo 145-8515, Japan, Phone: +81-3-3787-1151 Fax:+81-3-3787-4146, e-mail: suzubin@dent.showa-u.ac.jp 
